\title{
Capacidade de Retenção Hídrica do Estoque de Serapilheira de Eucalipto
}

\author{
Anne Francis Agostini Santos ${ }^{1}$, Antonio Carlos Pedro Carneiro ${ }^{2}$, \\ Diego Tyszka Martinez ${ }^{3}$, Sidney Fernando Caldeira ${ }^{3}$
}

${ }^{1}$ Departamento de Engenharia Florestal, Universidade do Estado de Mato Grosso - UNEMAT, Alta Floresta/MT, Brasil ${ }^{2}$ Empresa Matogrossense de Pesquisa, Assistência e Extensão Rural - EMPAER, Alta Floresta/MT, Brasil ${ }^{3}$ Departamento de Engenharia Florestal, Universidade Federal de Mato Grosso - UFMT, Cuiabá/MT, Brasil

\begin{abstract}
RESUMO
Objetivou-se avaliar o estoque e a retenção hídrica da serapilheira de 15 clones de espécies e de híbridos de Eucalyptus, aos três anos de idade. Foram coletadas amostras de $1 \mathrm{~m}^{2} \mathrm{da}$ serapilheira acumulada por repetição. Cada amostra foi separada em frações galhos, folhas e material amorfo. As frações foram secas em estufa de ventilação forçada até peso constante e, posteriormente, foi determinada sua massa seca. A capacidade de retenção hídrica foi determinada com a técnica estabelecida em 1955 por Blow. Não houve diferenças significativas em relação ao estoque de serapilheira, contudo a média de acúmulo foi de $4,96 \mathrm{Mg}^{-h a^{-1}}$, com variação entre 3,34 e 4,96 Mg.ha ${ }^{-1}$. As frações contribuíram na seguinte ordem decrescente: folha $>$ material amorfo > galhos. A capacidade de retenção hídrica (CRH) média foi de $249,58 \%$, tendo havido valor significativamente menor para os clones S-0208, S-0303 e S-0412. A frações contribuíram na seguinte ordem decrescente: material amorfo $>$ folhas $>$ galhos. Apesar da diferença significativa entre a CRH das frações, todas contribuem para a retenção hídrica total da serapilheira.
\end{abstract}

Palavras-chave: espécie exótica, acúmulo, umidade.

\section{Eucalyptus Litter Capacity of Stock and Water Retention}

\begin{abstract}
This research aimed to evaluate the stock and water retention of 15 litter clones and hybrids of the species Eucalyptus, with three years of age. Samples of $1 \mathrm{~m}^{2}$ of accumulated litter were collect by repetition. Each sample was separated into fractions consisting of twigs, leaves and amorphous material. The fractions were dried in a forced air kiln until they reached constant weight and, then the dry mass was determined. The water retention capacity was evaluate using the technique established by Blow in 1955. There were no significant differences reagrding litter stock, however, average accumulation was $4.96 \mathrm{Mg} \cdot \mathrm{ha}^{-1}$ and variation between 3.34 to $4.96 \mathrm{Mg}$.haFractions contributed in the following descending order: leaf $>$ amorphous material $>$ twigs. Water retention capacity average (WRC) was $249.58 \%$, the value was significantly lower to S-0208, S-0303 and S-0412 clones. The fractions contributed in decreasing order: amorphous material $>$ leaves $>$ twigs. Despite the significant difference between the fractions of WRC, all contribute to total water retention litter.
\end{abstract}

Keywords: exotic species, accumulation, moisture. 


\section{INTRODUÇÃO}

A camada superficial do solo em ambiente florestal, formada por folhas, galhos, órgãos reprodutivos e detritos, é conhecida como serapilheira e constitui uma importante via de equilíbrio ecológico e dinâmico nos ecossistemas florestais (Costa et al., 2010). Esse equilíbrio está relacionado à contribuição da serapilheira em relação ao ciclo da água e de nutrientes, que depende da constituição físico-química desse material.

Fisicamente, a serapilheira diminui o impacto das gotas de chuva sobre o solo e evita sua selagem, além de minimizar as taxas de evaporação (Vallejo, 1982), velocidade da enxurrada e, consequentemente, a redução do processo erosivo (Gonçalves et al., 2003). A umidade retida na serapilheira é liberada lenta e gradualmente no sistema ecológico, por meio dos diferentes estágios de decomposição que suas frações apresentam, e, dessa maneira, permite a manutenção da umidade superficial (Vallejo, 1982; Melos et al., 2010).

Essa dinâmica acontece pela decomposição do material senescente que altera o ambiente físico e químico, de formas direta e indireta (Facelli \& Pickett, 1991). Os mesmos autores relatam que a ação dos organismos degradadores libera nutrientes e substâncias fitotóxicas para o solo, que podem inibir o crescimento das raízes e plântulas, dificultando o alcance destas ao solo (Santos \& Válio, 2002). Com isso, a presença da serapilheira influencia o recrutamento de espécies na comunidade vegetal (Dzwonko \& Gawronski, 2002; Santos \& Válio, 2002). A serapilheira pode influir no estabelecimento das espécies em uma comunidade, por meio de sua espessura (Barrett, 1931), modificando a qualidade espectral da luz e a temperatura que ultrapassa o estoque de matéria morta sobre o solo (Facelli \& Pickett, 1991). Com isso, ambientes diferenciados são criados, gerando efeitos positivos e negativos para as espécies (Molofsky \& Augspurger, 1992).

Além disso, existe a ação de fungos patogênicos que, segundo Facelli et al. (1999), contribuem substancialmente para a mortalidade de plântulas quando há a presença da serapilheira. Wolkovich (2010) relatou que a presença dessa camada morta pode contribuir para a abundância de determinados artrópodes.

Estudos de serapilheira, que quantificam a produção e o estoque, contribuem para o conhecimento dos processos de retorno da matéria orgânica e nutrientes, da vegetação para o solo (Vital et al., 2004; Costa et al.,
2010; Schumacher et al., 2013), e, consequentemente, para a conservação da área estudada (Costa et al., 2010). Esse comportamento é dinâmico e sofre influência das condições meteorológicas da área, da época do ano e da idade das árvores (Piovesan et al., 2012).

Para espécies de rápido crescimento, como o Eucalyptus, essas análises são importantes para auxiliar a gestão dos sistemas de produção florestal (Ribeiro et al., 2002), bem como a sustentabilidade do solo, água e recursos nutricionais, que são muito exigidos em plantações de ciclo de produção curto (Bellote et al., 2008). Esse conhecimento adquirido pode promover a aplicação de práticas de manejo mais sustentáveis e auxiliar o silvicultor na escolha correta de técnicas mais eficazes de conservação e manutenção dos nutrientes (Schumacher et al., 2013). Assim, este trabalho teve o objetivo de avaliar a capacidade de retenção hídrica do estoque total e suas respectivas frações em 15 clones do gênero Eucalyptus plantados na Chapada dos Guimarães, em Mato Grosso.

\section{MATERIAL E MÉTODOS}

A área de estudo localiza-se no município de Chapada dos Guimarães (MT), cuja área está circunscrita à coordenada $15^{\circ} 21^{\prime} 01,02^{\prime \prime} \mathrm{S} ; 55^{\circ} 38^{\prime} 23,36^{\prime \prime} \mathrm{O}$, tomando-se por base um raio de $300 \mathrm{~m}$. O bioma original é o Cerrado (IBGE, 1992), com as fitofisionomias de mata semidecídua, mata ciliar, cerrado e cerradão.

A região apresenta clima tropical com estação seca de inverno, segundo a classificação de Köppen (1948). De acordo com Alvares et al. (2013), as médias anuais de temperatura variam entre $24 \mathrm{e} 26^{\circ} \mathrm{C}$, a precipitação pluviométrica média anual é $1.600 \mathrm{~mm}$, ocorrendo a maior precipitação em fevereiro $(218,3 \mathrm{~mm})$ e a menor em julho $(5,7 \mathrm{~mm})$.

O solo é classificado como Areia Quartzosa Álica (Vieira et al., 2011), a qual apresenta as seguintes características químicas: $\mathrm{pH}\left(\mathrm{H}_{2} \mathrm{O}\right)=5,6 ; \mathrm{P}=3,2 \mathrm{mg}^{-\mathrm{dm}^{-3}}$; $\mathrm{K}=24 \mathrm{mg} \cdot \mathrm{dm}^{-3} ; \mathrm{Ca}+\mathrm{Mg}=1,73 \mathrm{cmol}_{\mathrm{c}} \cdot \mathrm{dm}^{-3}$; $\mathrm{Ca}=0,96 \mathrm{cmol}_{c} \cdot \mathrm{dm}^{-3} ; \mathrm{Mg}=0,4 \mathrm{cmol}_{c} \cdot \mathrm{dm}^{-3} ; \mathrm{Al}=0,1 \mathrm{cmol}_{c} \cdot \mathrm{dm}^{-3} ;$ $\mathrm{H}=2,1 \mathrm{cmol} \cdot \mathrm{dm}^{-3} ;$ M.O. $=9,4 \mathrm{~g} \cdot \mathrm{dm}^{-3} ;(\mathrm{S})=1,47 \mathrm{cmol}_{c} \cdot \mathrm{dm}^{-3}$; $\mathrm{CTC}=3,64 \mathrm{cmol}_{c} \cdot \mathrm{dm}^{-3} ; \mathrm{V}=40,03 \%$. As características físicas do solo são: areia $=918 \mathrm{~g} \cdot \mathrm{kg}^{-1}$, argila $=55 \mathrm{~g} \cdot \mathrm{kg}^{-1}$ e silte $=27 \mathrm{~g} \cdot \mathrm{kg}^{-1}$.

Em julho de 2010, foram realizadas, em uma área de 2,64 hectares, a subsolagem por meio de um subsolador e a correção da acidez do solo com a aplicação de 
1,8 ton.ha ${ }^{-1}$ de calcário dolomítico. Após a incorporação, para cada hectare, o solo foi fertilizado com a aplicação de $115 \mathrm{~kg}$ de NPK 06:30:06 + 0,5\% Bórax, $400 \mathrm{~kg}$ de cloreto de potássio e $15 \mathrm{~kg}$ de boro gran a $10 \%$. O controle de plantas invasoras foi realizado com a aplicação combinada de herbicidas pré-emergentes para folhas estreita e larga, respectivamente, nas dosagens de 150 g.ha $^{-1}$ e 100 g.ha ${ }^{-1}$, nas linhas de plantio.

A implantação do experimento foi em agosto de 2010. O plantio foi constituído por 15 clones de Eucalyptus (Tabela 1). Os tratamentos (clone) foram dispostos em um talhão com 2,64 hectares de forma inteiramente casualizada. Não se efetuou replantio das árvores mortas. Cada tratamento foi arranjado em quatro parcelas (repetições) quadradas, sendo cada parcela estabelecida por 49 plantas com cada árvore em espaçamento de $3,60 \mathrm{~m} \times 2,50 \mathrm{~m}$, totalizando $441 \mathrm{~m}^{2}$. Para a coleta de dados, consideraram-se as 25 árvores centrais, sendo as demais consideradas bordadura.

Realizou-se a avaliação silvicultural dos clones de Eucalyptus em setembro de 2013, com 37 meses de plantio. As variáveis utilizadas foram a altura total, mensurada com o hipsômetro Blume Leiss, e o CAP, mensurado com o auxílio de fita métrica e, posteriormente, foi determinado o valor do diâmetro à altura do peito (DAP).

Tabela 1. Relação dos clones de espécies e de híbridos de Eucalyptus no plantio experimental na Chapada dos Guimarães (MT).

Table 1. List of clones of Eucalyptus belonging to different species and hybrids in experimental plantation at Chapada dos Guimarães, state of Mato Grosso, Brazil.

\begin{tabular}{cc|}
\hline Código do clone & Espécie ou híbrido \\
\hline S-0102 & E. urophylla \\
\hline S-0103 & E. urophylla $\times$ E. grandis \\
\hline S-0108 & E. urophylla \\
\hline S-0201 & E. urophylla \\
\hline S-0206 & E. urophylla \\
\hline S-0304 & E. urophylla \\
\hline S-0401 & E. camaldulensis \\
\hline S-0402 & E. urophylla $\times$ E. grandis \\
\hline S- 0403 & E. urophylla $\times$ E. grandis \\
\hline S-0406 & E. urophylla $\times$ E. camaldulensis \\
\hline S-0408 & E. urophylla $\times$ E. camaldulensis \\
\hline S-0119 & E. urophylla $\times$ E. camaldulensis \\
\hline S-0410 & E. urophylla $\times$ E. camaldulensis \\
\hline S-0411 & E. urophylla $\times$ E. camaldulensis \\
\hline S-0413 & E. camaldulensis $\times$ E. grandis \\
\hline
\end{tabular}

A camada de serapilheira estocada sobre a superfície do solo foi coletada no final do período seco (setembro de 2013), por meio de um gabarito de madeira $(1 \mathrm{~m} \times 1 \mathrm{~m})$, em cada repetição. Tal material foi acondicionado em sacos de aniagem e conduzido ao laboratório. Posteriormente, partículas de solo foram removidas sobre peneira (Scoriza et al., 2012) e a serapilheira foi triada nas seguintes frações: galhos, folhas, órgãos reprodutivos e material amorfo. Porém, na composição da serapilheira, a fração de material reprodutivo não foi observada em qualquer uma das amostras, por se tratar de povoamento jovem. Em seguida, as frações foram secas em estufa de circulação forçada de ar a $70^{\circ} \mathrm{C}$, até atingirem massa seca constante, que foi determinada em balança de precisão $0,01 \mathrm{~g}$.

A capacidade de retenção hídrica (CRH) foi avaliada pelo método desenvolvido por Blow (1955), de acordo com a Equação 1. Cada fração foi reidratada por imersão em água durante 90 minutos e, em seguida, depositada em peneiras durante 30 minutos para o escoamento da água superficial, com posterior determinação da massa úmida $(\mathrm{Mu})$ em balança de precisão 0,01 g. Novamente, as frações foram secas em estufa de circulação forçada a $70^{\circ} \mathrm{C}$, até o estado anidro, e novamente pesadas para determinar suas massas secas (Ms).

Para determinar a capacidade de retenção hídrica (CRH\%), utilizou-se a seguinte Equação 1:

$\mathrm{CRH}(\%)=\left[\frac{(\mathrm{Mu}-\mathrm{Ms})}{\mathrm{MS}}\right] \times 100$

Sendo CRH (\%): capacidade de retenção hídrica; Mu: massa úmida; Ms: massa seca.

Os dados foram submetidos ao teste de Lilliefors e Cochran e, quando necessário, transformados pela expressão $\sqrt{ } \mathrm{x}$. Após efetuada a análise de variância, os tratamentos foram comparados pelo teste de Tukey a 5\% e a $1 \%$. Complementarmente, realizou-se a correlação de Pearson entre as variáveis altura total e DAP, além do estoque de serapilheira total e suas frações, como também da capacidade de retenção hídrica.

\section{RESULTADOS E DISCUSSÃO}

Aos 3 anos de idade, a sobrevivência média dos clones foi de $96,4 \%$, variando de $84 \%$ a $100 \%$. As médias para a altura total e DAP foram, respectivamente, $13,7 \mathrm{~m} \pm 0,44 \mathrm{~m}$ e $14,9 \mathrm{~cm} \pm 0,78 \mathrm{~cm}$, com baixos 
valores para os coeficientes de variação, 3,20\% e 5,25\%, respectivamente.

Houve diferenças significativas sobre a massa média total do estoque de serapilheira dos diferentes clones de Eucalyptus $\left(\mathrm{F}=2,36^{*}\right)$, entre as frações destes $\left(F=2,35^{\star *}\right)$ e também dentro das frações $\left(57,97^{* *}\right)$, além de haver interação entre fatores (clones $\mathrm{x}$ frações) $\left(\mathrm{F}=3,45^{\star *}\right)$ sobre a massa média do estoque de serapilheira (Tabela 2).

$\mathrm{O}$ estoque médio total de serapilheira foi de 4,96 Mg.ha' ${ }^{-1}$ e variou de 3,34 Mg.ha' ${ }^{-1}$ a 6,43 Mg.ha ${ }^{-1}$ (Tabela 2). Essa variação existiu também em Demessie et al. (2012) para a serapilheira de povoamentos de Eucalyptus saligna, E. camaldulensis e E. pellita, com 22 a 30 anos, cujo acúmulo médio foi de 11,6 Mg.ha- ${ }^{-1}$ e variou de 9,7 a 12,6 Mg.ha- ${ }^{-1}$. Por outro lado, Zaia
\& Gama-Rodrigues (2004) registraram média de 6,69 Mg.ha' ${ }^{-1}$ e variação entre 3,89 e 8,21 Mg.ha ${ }^{-1}$ para E. grandis, E. camaldulensis e E. pellita, no verão, aos 6 anos de idade.

Ao comparar o estoque total entre os clones, a quantidade de serapilheira estocada foi semelhante, para a maioria dos materiais testados, com superioridade para os clones S-0410 e S-0411, respectivamente 6,43 Mg.ha- e 6,24 Mg.ha' ${ }^{-1}$, apenas em relação aos clones S-0102 e S-0304, 3,34 Mg.ha- e 3,51 Mg.harespectivamente (Tabela 2).

O acúmulo de serapilheira é variável e depende da espécie, da idade, do sítio e da disponibilidade de nutrientes no solo (Selle, 2007). Há registros com espécies de Eucalyptus com deposição 6,33 Mg.ha ${ }^{-1}$ aos 14 anos de idade em Minas Gerais (Souza \&

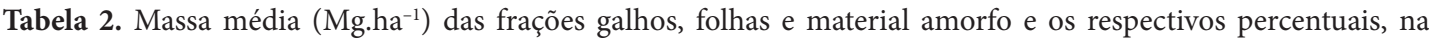
composição do estoque de serapilheira de clones das espécies e híbridos de Eucalyptus plantados na Chapada dos Guimarães (MT).

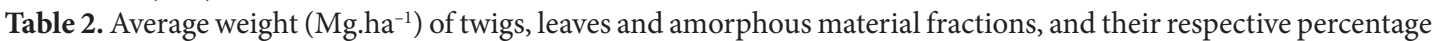
in the stock composition of litter clones species and hybrids Eucalyptus, planted in Chapada dos Guimarães, Mato Grosso, Brazil.

\begin{tabular}{|c|c|c|c|c|c|c|c|}
\hline \multirow{2}{*}{ Clones } & \multirow{2}{*}{$\frac{\text { Total }}{\text { Mg.ha }^{-1}}$} & \multicolumn{2}{|c|}{ Galhos } & \multicolumn{2}{|c|}{ Folhas } & \multicolumn{2}{|c|}{ Amorfo } \\
\hline & & Mg.ha $^{-1}$ & $\%$ & Mg.ha $^{-1}$ & $\%$ & Mg.ha $^{-1}$ & $\%$ \\
\hline S-0102 & $3,34 b$ & $0,60 \mathrm{aB}$ & 22,14 & $1,67 \mathrm{abcA}$ & 47,44 & $1,07 \mathrm{cAB}$ & 30,41 \\
\hline S-0103 & $4,35 \mathrm{ab}$ & $0,78 \mathrm{aB}$ & 17,92 & $2,30 \mathrm{abA}$ & 52,87 & $1,27 \mathrm{abcAB}$ & 29,20 \\
\hline S-0108 & $4,48 \mathrm{ab}$ & $1,23 \mathrm{aA}$ & 27,43 & $1,75 \mathrm{abcA}$ & 39,16 & $1,49 \mathrm{abcA}$ & 33,39 \\
\hline S-0201 & $5,51 \mathrm{ab}$ & $0,78 \mathrm{aB}$ & 15,99 & $1,81 \mathrm{abcA}$ & 37,22 & $2,28 \mathrm{abcA}$ & 46,78 \\
\hline S-0206 & $5,26 \mathrm{ab}$ & $0,73 \mathrm{aC}$ & 13,94 & $2,91 \mathrm{abA}$ & 55,40 & $1,61 \mathrm{abcB}$ & 30,65 \\
\hline S-0208 & $4,85 \mathrm{ab}$ & $0,75 \mathrm{aB}$ & 15,64 & $2,56 \mathrm{abA}$ & 52,96 & $1,52 \mathrm{abcAB}$ & 31,39 \\
\hline S-0302 & $5,15 \mathrm{ab}$ & $1,60 \mathrm{aAB}$ & 31,06 & $2,52 \mathrm{abA}$ & 48,89 & $1,03 \mathrm{cB}$ & 20,03 \\
\hline S-0304 & $3,51 \mathrm{~b}$ & $1,00 \mathrm{aB}$ & 28,52 & $0,54 \mathrm{cB}$ & 15,55 & $1,96 \mathrm{abcA}$ & 55,91 \\
\hline S-0401 & $4,73 \mathrm{ab}$ & $0,94 \mathrm{aB}$ & 19,86 & $2,11 \mathrm{abA}$ & 44,64 & $1,68 \mathrm{abcA}$ & 35,48 \\
\hline S-0402 & $5,41 \mathrm{ab}$ & $1,77 \mathrm{aAB}$ & 32,69 & $2,56 \mathrm{abA}$ & 47,39 & $1,07 \mathrm{bcB}$ & 19,91 \\
\hline S- 0403 & $4,78 \mathrm{ab}$ & $1,73 \mathrm{aA}$ & 36,25 & $1,59 \mathrm{abcA}$ & 33,41 & $1,45 \mathrm{abcA}$ & 30,32 \\
\hline S-0406 & $4,53 \mathrm{ab}$ & $0,77 \mathrm{aB}$ & 17,02 & $1,42 \mathrm{bcAB}$ & 31,57 & $2,32 \mathrm{abcA}$ & 51,40 \\
\hline S-0408 & $5,28 \mathrm{ab}$ & $1,13 \mathrm{aB}$ & 21,40 & $1,54 \mathrm{bcAB}$ & 29,22 & $2,60 \mathrm{abA}$ & 49,37 \\
\hline S-0119 & $5,09 \mathrm{ab}$ & 1,16 aA & 22,78 & $1,94 \mathrm{abcA}$ & 38,18 & $1,98 \mathrm{abcA}$ & 39,02 \\
\hline S-0410 & $6,43 \mathrm{a}$ & $1,28 \mathrm{aB}$ & 19,93 & $3,07 \mathrm{aA}$ & 47,84 & $2,07 \mathrm{abcAB}$ & 32,21 \\
\hline S-0411 & $6,24 \mathrm{a}$ & $1,42 \mathrm{aB}$ & 22,82 & $1,79 \mathrm{abcB}$ & 28,81 & $3,01 \mathrm{aA}$ & 48,35 \\
\hline S-0412 & $5,46 \mathrm{ab}$ & $0,93 \mathrm{aB}$ & 17,15 & $2,37 \mathrm{abA}$ & 43,43 & $2,15 \mathrm{abcA}$ & 39,40 \\
\hline S-0413 & $4,90 \mathrm{ab}$ & $0,82 \mathrm{aB}$ & 16,86 & $2,68 \mathrm{abA}$ & 54,78 & $1,39 \mathrm{abcB}$ & 28,34 \\
\hline Média & 4,96 & $1,09 \mathrm{C}$ & 22,19 & $2,06 \mathrm{~A}$ & 41,60 & 1,77 B & 36,20 \\
\hline $\mathrm{CV}_{1}(\%)$ & 20,45 & & & CV ( & 9,56 & & \\
\hline F1 & $2,36^{*}$ & & & & & & \\
\hline $\mathrm{F} 2$ & & & & & & & \\
\hline F1xF2 & & & & & & & \\
\hline
\end{tabular}

Valores seguidos pelas mesmas letras minúscula na coluna e maiúscula na linha não diferem estatisticamente entre si. Foi aplicado o Teste de Tukey ao nível de 5\% de probabilidade. F1: F calculada para clones; F2: F calculada entre frações; CV\%: coeficiente de variação; M: massa. ${ }^{*}$ significativo a $5 \%$ de probabilidade; ${ }^{* *}$ significativo a $1 \%$ de probabilidade. 
Davide, 2001) e, no Rio de Janeiro, de 16,6 Mg.ha ${ }^{-1}$, aos 7 anos de idade (Balieiro et al., 2004), e também de 2,2 Mg.ha ${ }^{-1}$, no segundo ano de estabelecimento (Melos et al., 2010).

O estoque de biomassa morta por espécies de Eucalyptus é superior ao de muitos plantios comerciais com outras espécies. Souza \& Davide (2001) detectaram que E. saligna produziu 2,5 vezes mais que Bracatinga (Mimosa scabrella) e Demessie et al. (2012) registraram que E. saligna, E. camaldulensis e E. globulus produziram o dobro das coníferas Cupressus lusitanica, Pinus patula e Jeniperus procera, no sudoeste da Etiópia.

O maior estoque de serapilheira de Eucalyptus provavelmente está relacionado com os baixos coeficiente de decomposição desse material, que variam de 0,54 a 1,0 (Zaia \& Gama-Rodrigues, 2004; Schumacher et al., 2013;). Em florestas tropicais, as taxas de decomposição são maiores do que 1,0 (Pires et al., 2006). A lenta decomposição da serapilheira de eucaliptos é reflexo da elevada concentração de lignina nos tecidos foliares (Schumacher et al., 2013), que não favorecem os organismos edáficos decompositores, bem como do microclima desfavorável a esse processo, que ocorre nos plantios de espécies desse gênero, como altas temperaturas e baixa umidade (Melos et al., 2010).

A lenta decomposição da serapilheira de Eucalyptus pode ser benéfica (Souza \& Davide, 2001), pois o acúmulo de serapilheira sobre a superfície do solo estoca nutrientes para futuras mineralizações e ciclagem (Cunha et al., 2013). Essa dinâmica mantém a fertilidade e auxilia na manutenção do ecossistema para as plantas (Mateus et al., 2013) a longo prazo.

A média geral para todos os clones foi de 4,96, Mg.ha ${ }^{-1}$. Entre as frações da serapilheira, houve diferença significativa, sendo a maior contribuição a fração folha com 2,06 Mg.ha-1 ${ }^{-1}(41,60 \%$ da serapilheira total) e a menor contribuição, a fração galho com 1,09 Mg.ha-1 (22,19\% da serapilheira total), além da fração amorfo com 1,77 Mg.ha ${ }^{-1}$ (36,20\% da serapilheira total) (Tabela 2).

Da mesma maneira que no presente trabalho, outros autores também verificaram que, em povoamentos de eucalipto entre 6,0 e 7,5 anos, a fração folha apresentou maiores contribuições no estoque de serapilheira, mas, diferentemente, a fração galho apresentou maior contribuição do que a amorfo (Zaia \& Gama-Rodrigues, 2004; Schumacher et al., 2013). Acredita-se que a divergência foi influenciada pela idade das plantas de eucalipto neste trabalho (três anos de estabelecimento), uma vez que, segundo Cunha et al. (2013), do terceiro ao quinto ano aumenta a desrama natural, com consequente incremento da quantidade de galhos na serapilheira.

Para diversas espécies, mais de 70\% da serapilheira é composta de folhas, sendo tal predominância dependente da idade do povoamento, pois, quanto mais velho, maior a produção de galhos finos e grossos, diminuindo a porcentagem de folhas (Reis \& Barros, 1990). Porém, em plantios mais velhos, a porcentagem de folhas ainda é expressiva (de $60 \%$ a $80 \%$ do valor da biomassa morta depositada sobre o solo (Schumacher et al., 2013).

Em geral, aos 37 meses de estabelecimento, avaliados na estação seca, em Chapada dos Guimarães, os clones que se destacaram foram S-0410 e S-0411, com 6,43 e 6,24 Mg.ha ${ }^{-1}$, respectivamente. Essa superioridade em relação aos demais está no fato de que o clone S-0410 apresentou um dos maiores estoques na fração folha com 3,07 Mg.ha ${ }^{-1}$ e o clone S-0411, com 3,01 Mg.ha ${ }^{-1}$ na fração amorfo. A representação da fração galho foi semelhante em todos os materiais testados.

Houve diferenças significativas na capacidade de retenção hídrica total da serapilheira (CRH) do clone de Eucalyptus ( $\left.\mathrm{F}=26,44^{\star *}\right)$, como também entre as frações destes $\left(\mathrm{F}=32,95^{\star *}\right)$, dentro das frações $\left(566,31^{* *}\right)$. Ainda foi registrada interação entre esses fatores (clones $x$ frações) $\left(F=4,99^{* *}\right)$ (Tabela 3).

A capacidade de retenção hídrica total da serapilheira (CRH) dos clones de Eucalyptus foi, em média, 249,58\% (Tabela 3). Na literatura, foram encontrados valores de CRH próximos à média do presente trabalho. Em Blow (1955), que pesquisou a serapilheira de florestas de carvalho, os percentuais foram de $200 \%$ a $250 \%$. Melos et al. (2010) obtiveram 235\% em um plantio com híbrido de E. urophylla x E. grandis e Mateus et al. (2013) encontraram $230 \%$ para pastagem perturbada e abandonada e $206 \%$ em fragmento florestal nativo.

Entretanto, Vallejo (1982) registrou retenção superior a $300 \%$ para serapilheira de floresta latifoliada, enquanto Miranda (1992) observou apenas 200\% de retenção de floresta ombrófila. A capacidade de retenção hídrica da serapilheira das espécies e híbridos de Eucalyptus é próxima à de florestas naturais, mesmo com composição do material orgânico mais homogênea (Melos et al., 2010).

Ao se comparar a CRH do estoque de serapilheira total entre os clones testados, o maior valor foi registrado para o clone S-0201, com 334,01\%, contudo foi semelhante 
Tabela 3. Capacidade de retenção hídrica (\%) das frações galhos, folhas e material amorfo na composição da serapilheira de clones de espécies e híbridos de Eucalyptus plantados na Chapada dos Guimarães (MT).

Table 3. Water Retention Capacity (\%) of twig, leaves and amorphous material fractions in the composition of litter clones species and hybrids Eucalyptus, Chapada dos Guimarães, Mato Grosso, Brazil.

\begin{tabular}{|c|c|c|c|c|}
\hline \multirow{2}{*}{ Clones } & \multicolumn{4}{|c|}{ Capacidade de retenção hídrica (\%) } \\
\hline & Total & Galhos & Folhas & Amorfo \\
\hline S-0102 & $238,32 \mathrm{~b}$ & $52,20 \mathrm{aB}$ & $67,32 \mathrm{deB}$ & $118,79 \mathrm{abA}$ \\
\hline S-0103 & $243,83 \mathrm{~b}$ & $37,00 \mathrm{abcB}$ & $95,32 \mathrm{cdA}$ & $104,67 \mathrm{abA}$ \\
\hline S-0108 & $278,75 \mathrm{ab}$ & $40,96 \mathrm{abcB}$ & $121,12 \mathrm{abcA}$ & $116,67 \mathrm{abA}$ \\
\hline S-0201 & $334,01 \mathrm{a}$ & $56,56 \mathrm{aB}$ & $143,38 \mathrm{aA}$ & $134,06 \mathrm{aA}$ \\
\hline S-0206 & $281,38 \mathrm{ab}$ & $42,37 \mathrm{abcB}$ & $118,49 \mathrm{abcA}$ & $120,50 \mathrm{abA}$ \\
\hline S-0208 & $88,65 \mathrm{c}$ & $10,15 \mathrm{cB}$ & $29,89 \mathrm{fAB}$ & $48,61 \mathrm{dA}$ \\
\hline S-0302 & $85,43 \mathrm{c}$ & $12,93 \mathrm{bcB}$ & $17,31 \mathrm{fB}$ & $55,17 \mathrm{cdA}$ \\
\hline S-0304 & $265,35 \mathrm{ab}$ & $52,58 \mathrm{aB}$ & $107,92 \mathrm{abcA}$ & $104,83 \mathrm{abA}$ \\
\hline S-0401 & $289,24 \mathrm{ab}$ & $34,67 \mathrm{abcB}$ & $120,93 \mathrm{abcA}$ & $133,63 \mathrm{aA}$ \\
\hline S-0402 & $259,10 \mathrm{~b}$ & $34,6 \mathrm{abcB}$ & $107,36 \mathrm{bcA}$ & $117,13 \mathrm{abA}$ \\
\hline S- 0403 & $292,57 \mathrm{ab}$ & $44,01 \mathrm{abB}$ & $118,61 \mathrm{abcA}$ & $129,94 \mathrm{aA}$ \\
\hline S-0406 & $291,52 \mathrm{ab}$ & $46,49 \mathrm{abB}$ & $118,49 \mathrm{abcA}$ & $126,52 \mathrm{aA}$ \\
\hline S-0408 & $266,60 \mathrm{ab}$ & $32,62 \mathrm{abcB}$ & $113,77 \mathrm{abcA}$ & $120,2 \mathrm{abA}$ \\
\hline S-0119 & $277,49 a b$ & $38,41 \mathrm{abcB}$ & $130,83 \mathrm{abcA}$ & $108,24 \mathrm{abA}$ \\
\hline S-0410 & $284,98 \mathrm{ab}$ & $41,18 \mathrm{abcB}$ & $137,91 \mathrm{abA}$ & $105,88 \mathrm{abB}$ \\
\hline S-0411 & $302,64 a b$ & $42,02 \mathrm{abcB}$ & $128,91 \mathrm{abcA}$ & $131,7 \mathrm{aA}$ \\
\hline S-0412 & $138,01 \mathrm{c}$ & $9,95 \mathrm{cC}$ & $39,31 \mathrm{efB}$ & $88,74 \mathrm{bcA}$ \\
\hline S-0413 & $274,64 \mathrm{ab}$ & $37,72 \mathrm{abcB}$ & $117,12 \mathrm{abcA}$ & $119,79 \mathrm{abA}$ \\
\hline Media & 249,58 & $37,06 \mathrm{C}$ & $101,92 \mathrm{~B}$ & $110,28 \mathrm{~A}$ \\
\hline CV (\%) & 11,07 & & CV (\%) 17,20 & \\
\hline F1 & $26,44^{* *}$ & & $32,95^{* *}$ & \\
\hline $\mathrm{F} 2$ & & & $566,31^{* *}$ & \\
\hline F1XF2 & & & $4,99^{* *}$ & \\
\hline
\end{tabular}

As médias seguidas pelas mesmas letras minúscula nas colunas e maiúscula nas linhas não diferem estatisticamente entre si. Foi aplicado o Teste de Tukey ao nível de 5\% de probabilidade. F1: F calculada para clones; F2: F calculada entre frações. CV\%: coeficiente de variação. ${ }^{* *}$ significativo a $1 \%$ de probabilidade.

ao de outros 11 clones (Tabela 3). Os menores foram para os clones S-0208, S-0302 e S-0412, com 88,65\%, $85,43 \%$ e $138,01 \%$ de retenção hídrica, respectivamente (Tabela 3), destacando-se a variação existente na $\mathrm{CRH}$ dos diferentes clones de Eucalyptus. Para Vallejo (1982), a CRH não depende apenas da quantidade de material orgânico depositado, mas também do grau de decomposição das frações. De acordo com esse autor, camadas do estoque de serapilheira com maior grau de decomposição apresentam maior superfície específica e, dessa maneira, seu potencial de retenção hídrica é maior quando comparado ao de camadas mais superficiais e não decompostas.

Esse fato pode ser observado no clone S-0201, com um dos destaques em CRH, tendo a fração amorfo desse material retido $134,06 \%$ de umidade, enquanto os materiais mais inferiores (S-0208, S-0302 e S-0412) absorveram $48,61 \%, 55,17 \%$ e $88,74 \%$, respectivamente.

Ao comparar as diferentes frações de serapilheira, a fração folha apresentou menor capacidade de retenção hídrica do que a amorfo (Tabela 3 ). Tal fato pode ser compreendido pela menor adesão superficial, cuja influência depende da área de foliar, relevo, forma, grau de decomposição e relação superfície/peso (Mateus et al., 2013). Na fração galho, isso ocorre devido à sua natureza, cujo material lenhoso apresenta menor absorção de umidade, sendo mais favorável a canalização ou o escoamento superficial hídrico (Montezuma, 2005). Por outro lado, de maneira geral, não houve diferenças significativas na capacidade de retenção hídrica, na comparação entre as frações folhas e material amorfo (Tabela 3). 
Tabela 4. Correlação de Pearson entre as variáveis altura total $(\mathrm{Ht})$, diâmetro à altura do peito (DAP), e as massas acumuladas de serapilheira, total (Mt) e das frações galho (Mg), folha (Mf) e material amorfo (Mma), e a capacidade de retenção hídrica, total (CRHt) e das frações galho (CRHg), folha (CRHf) e material amorfo (CRHma), para clones de espécies e de híbridos de Eucalyptus plantados na Chapada dos Guimarães (MT).

Table 4. Pearson correlation among variables total height (Ht), Diameter at chest height (DAP), and accumulated masses litter, total (Mt) and twig (Mg), leaf (Mf) and amorphous material fractions (Mma), and water retention capacity, total (CRHt) and twig (CRHg) leaf (CRHf) and amorphous material fractions (CRHma) for clones species and hybrids Eucalyptus, planted in Chapada dos Guimarães, Mato Grosso, Brazil.

\begin{tabular}{|c|c|c|c|c|c|c|c|c|c|c|}
\hline Variável & $\begin{array}{c}\mathrm{Ht} \\
\mathrm{m}\end{array}$ & $\begin{array}{c}\text { DAP } \\
\mathrm{cm}\end{array}$ & $\begin{array}{c}\text { Mt } \\
\text { Mg.ha }^{-1}\end{array}$ & $\begin{array}{c}\text { Mg } \\
\mathrm{Mg} \cdot \mathrm{ha}^{-1}\end{array}$ & $\begin{array}{c}\text { Mf } \\
\text { Mg.ha }^{-1}\end{array}$ & $\begin{array}{c}\text { Mma } \\
\text { Mg.ha }^{-1}\end{array}$ & $\begin{array}{c}\text { CRHt } \\
\%\end{array}$ & $\begin{array}{c}\text { CRHg } \\
\%\end{array}$ & $\begin{array}{c}\text { CRHf } \\
\%\end{array}$ & $\begin{array}{c}\text { CRHma } \\
\%\end{array}$ \\
\hline $\mathrm{Ht}$ & 1,0 & - & - & - & - & - & - & - & - & - \\
\hline DAP & $0,3^{\star *}$ & 1,0 & - & - & - & - & - & - & - & - \\
\hline Mt & $0,30^{\mathrm{ns}}$ & $0,09^{\text {ns }}$ & 1,0 & - & - & - & - & - & - & - \\
\hline $\mathrm{Mg}$ & $0,4^{\mathrm{ns}}$ & $0,23^{\text {ns }}$ & $0,40^{\mathrm{ns}}$ & 1,0 & - & - & - & - & - & - \\
\hline Mf & $-0,14^{\mathrm{ns}}$ & $-0,02^{\mathrm{ns}}$ & $0,55^{\star}$ & $0,03^{\text {ns }}$ & 1,0 & - & - & - & - & - \\
\hline Mma & $0,30^{\mathrm{ns}}$ & $0,08^{\text {ns }}$ & $0,46^{\mathrm{ns}}$ & $-0,06^{\mathrm{ns}}$ & $-0,32^{\mathrm{ns}}$ & 1,0 & - & - & - & - \\
\hline CRHt & $0,09^{\text {ns }}$ & $-0,22^{\mathrm{ns}}$ & $0,07^{\mathrm{ns}}$ & $-0,01^{\mathrm{ns}}$ & $-0,30^{\mathrm{ns}}$ & $0,36^{\mathrm{ns}}$ & 1,0 & - & - & - \\
\hline $\mathrm{CRHg}$ & $0,18^{\text {ns }}$ & $-0,14^{\mathrm{ns}}$ & $-0,25^{\mathrm{ns}}$ & $-0,15^{\mathrm{ns}}$ & $-0,48^{*}$ & $0,18^{\mathrm{ns}}$ & $0,86^{* *}$ & 1,0 & - & - \\
\hline CRHf & $0,06^{\mathrm{ns}}$ & $-0,25^{\mathrm{ns}}$ & $0,22^{\text {ns }}$ & $0,06^{\mathrm{ns}}$ & $-0,19^{\mathrm{ns}}$ & $0,42^{\mathrm{ns}}$ & $0,96^{\star *}$ & $0,77^{\star *}$ & 1,0 & - \\
\hline CRHma & $0,08^{\text {ns }}$ & $-0,16^{\mathrm{ns}}$ & $0,02^{\text {ns }}$ & $-0,02^{\mathrm{ns}}$ & $-0,32^{\text {ns }}$ & $0,33^{\text {ns }}$ & $0,93^{* *}$ & $0,78^{* *}$ & $0,84^{* *}$ & 1,0 \\
\hline
\end{tabular}

ns: não significativo; ${ }^{*}$ significativo a $5 \%$ de probabilidade; ${ }^{* *}$ significativo a $1 \%$ de probabilidade.

Não foram registradas correlações significativas entre os valores de crescimentos primário e secundário, respectivamente, Ht e DAP, com a massa total de serapilheira, de suas frações, com a CRH total ou de suas frações (Tabela 4). Contudo, para a massa total de serapilheira, foi registrada correlação de 0,55 , devido à massa de folhas, provavelmente pela maior contribuição desta na serapilheira.

Não se observaram correlações significativas entre a massa do estoque de serapilheira total e a $\mathrm{CRH}$ do estoque total e das frações do estoque de serapilheira (Tabela 4). As correlações foram significativas na combinação da CRH do estoque de serapilheira total com todas as frações e também de todas as frações entre si (Tabela 4). Esse fato indicou que todas as frações contribuem efetivamente para o volume hídrico total retido no estoque de serapilheira dos clones.

\section{CONCLUSÕES}

Em geral, não houve diferenças entre os clones de eucalipto em relação ao estoque de serapilheira total e suas frações.

Para a média dos clones, a ordem decrescente da contribuição das frações na composição do estoque de serapilheira foi a seguinte: folhas $>$ material amorfo $>$ galhos .
A capacidade de retenção hídrica média do estoque total da serapilheira dos clones foi de $249,58 \%$, com valor significativamente menor para os clones S-0208, S-0302 e S-0412.

Para a média dos clones, a ordem decrescente da capacidade de retenção hídrica das frações foi a seguinte: material amorfo $>$ folhas $>$ galhos

Todas as frações contribuíram efetivamente para a capacidade de retenção hídrica do estoque total de serapilheira.

Não houve correlações significativas entre a massa total de serapilheira e de suas frações com a CRH da serapilheira total e das respectivas frações.

\section{STATUS DA SUBMISSÃO}

Recebido: 5 set., 2016

Aceito: 16 set., 2016

\section{AUTOR(ES) PARA CORRESPONDÊNCIA}

\section{Anne Francis Agostini Santos}

Departamento de Engenharia Florestal, Universidade do Estado de Mato Grosso UNEMAT, Avenida Perimetral Rogério Silva, s/n, Jardim Flamboyant, CEP 78580-000, Alta Floresta, MT, Brasil e-mail: annef_168@hotmail.com 


\section{REFERENNCIAS}

Alvares CA, Stape JL, Sentelhas PC, Gonçalves JLM, Sparovek G. Köppen's climate classification map for Brazil. Meteorologische Zeitschrift 2013; 711-728.

Balieiro FC, Franco AA, Pereira MG, Campello EFC, Dias LE, Faria SM et al. Dinâmica da serapilheira e transferência de nitrogênio ao solo, em plantios de Pseudosamanea guachapele e Eucalyptus grandis. Pesquisa Agropecuaria Brasileira 2004; 39(6): 597-601. http://dx.doi.org/10.1590/ S0100-204X2004000600012.

Barrett LI. Influence of forest litter on the germination and early survival of chestnut oak, Quercus montana Willd. Ecology 1931; 12(3): 476-484. http://dx.doi. org/10.2307/1928993.

Bellote AFJ, Dedecek RA, Silva HD. Nutrientes minerais, biomassa e deposição de serapilheira em plantio de Eucalyptus com diferentes sistemas de manejo de resíduos florestais. Pesquisa Florestal Brasileira 2008; 56: 31-41.

Blow FE. Quantity and hydrologic characteristics of litter under upland oak forests in Eastern Tennessee. Journal of Forestry 1955; 53: 190-195.

Costa CCA, Camacho RGV, Macedo ID, Silva PCM. Análise comparativa da produção de serapilheira em fragmentos arbóreos e arbustivos em área de caatinga na Flona de Açu - RN. Revista Árvore 2010; 2(34): 259-265. http://dx.doi.org/10.1590/S0100-67622010000200008.

Cunha FV No, Teles PSS, Pereira MG, Bellumath VGH, Alonson JM. Acúmulo e decomposição da serapilheira em quatro formações florestais. Ciência Florestal 2013; 23(3): 379-387. http://dx.doi.org/10.5902/1980509810549.

Demessie A, Singh BR, Lal R, Strand LT. Leaf litter fall and litter decomposition under Eucalyptus and coniferous plantation in Gambo District, southern Ethiopia. Acta Agricultura e Scandinavica Section B: Soil and Plant Science 2012; 62: 467-476.

Dzwonko Z, Gawronski S. Influence of litter and weather on seedling recruitment in a mixed oak-pine woodland. Annals of Botany 2002; 90(2): 245-251. PMid:12197522. http://dx.doi.org/10.1093/aob/mcf178.

Facelli JM, Pickett STA. Plant litter: its dynamics and effects on plant community structure. Botanical Review 1991; 57(1): 1-32. http://dx.doi.org/10.1007/BF02858763.

Facelli JM, Williams R, Fricker S, Ladd B. Establishment and growth of seedlings of Eucalyptus obliqua: interactive effects of litter, water, and pathogens. Australian Journal of Ecology 1999; 24(5): 484-494. http://dx.doi.org/10.1046/ j.1440-169x.1999.00988.x.

Gonçalves JLM, Nogueira LR, Ducatti F. Recuperação de solos degradados. In: Kageyama PY, Oliveira RE, Moraes LFD, Engel VL, Gandara FB, editores. Restauração ecológica de ecossistemas naturais. Botucatu: FEPAF; 2003.
Instituto Brasileiro de Geografia e Estatística - IBGE. Classificação da vegetação brasileira, adaptada a um sistema universal. Rio de Janeiro: IBGE; 1992.

Köppen W. Climatologia: con un estudio de los climas de la tierra. México: Fondo de Cultura Econômica; 1948.

Mateus FA, Miranda CC, Valcarcel R, Figueiredo PHA. Estoque e capacidade de retenção hídrica da serrapilheira acumulada na restauração florestal de áreas perturbadas na Mata Atlântica. Floresta e Ambiente 2013; 20(3): 336-343.

Melos ARM, Sato AM, Coelho AL No. Produção, estoque e retenção hídrica da serrapilheira em encosta sob plantio de híbridos de Eucalyptus urophylla e Eucalyptus grandis: médio vale do Rio Paraíba do Sul. Anuário do Instituto de Geociências 2010; 33(2): 66-73.

Miranda JC. Interceptação das chuvas pela vegetação florestal e serrapilheira nas encostas do Maciço da Tijuca: Parque Nacional da Tijuca, RJ [dissertação]. Rio de Janeiro: Universidade Federal do Rio de Janeiro; 1992.

Molofsky J, Augspurger CK. The effect of leaf litter on early seedling establishment in a tropical forest. Ecology 1992; 73(1): 68-77. http://dx.doi.org/10.2307/1938721.

Montezuma RCM. Produção e reabilitação funcional do piso florestal em clareira de deslizamento: Parque Nacional da tijuca, Rio de Janeiro. Anuário do Instituto de Geociências 2005; 28(2): 155-156.

Piovesan G, Schumacher MV, Viera M, Lopes VGWC. Deposição de serapilheira em povoamentos de pinus. Pesquisa Agropecuária Tropical 2012; 42(2): 206-211. http://dx.doi.org/10.1590/S1983-40632012000200012.

Pires LA, Britez RM, Martel G, Pagano SN. Produção, acúmulo e decomposição da serapilheira em uma restinga da Ilha do Mel, Paranaguá, PR, Brasil. Acta Botanica Brasílica 2006; 20(1): 173-1846. http://dx.doi.org/10.1590/ S0102-33062006000100016.

Reis MG, Barros NF. Ciclagem de nutrientes em plantios de Eucalipto. In: Barros NF, Novais RF, editores. Relação solo-eucalipto. Viçosa: UFV; 1990.

Ribeiro C, Madeira M, Araújo MC. Decomposition and nutrient release from leaf litter of Eucalyptus globulus grown under different water and nutrient regimes. Forest Ecology and Management 2002; 171(1-2): 31-41. http:// dx.doi.org/10.1016/S0378-1127(02)00459-0.

Santos SL, Válio IFM. Litter accumulation and its effect on seedling recruitment in a Southeast Brazilian Tropical Forest. Revista Brasileira de Botanica = Brazilian Journal of Botany 2002; 25(1): 89-92. http://dx.doi.org/10.1590/ S0100-84042002000100011.

Schumacher MV, Corrêa RS, Viera M, Araújo EF. Produção e decomposição de serapilheira em um povoamento de Eucalyptus urophylla $x$ Eucalyptus globulus maidenii. Cerne 2013; 19(3): 501-508. http://dx.doi.org/10.1590/ S0104-77602013000300018. 
Scoriza, RN, Pereira, MP, Pereira, GHA, Machado, DL, Silva, EMR. Métodos para coleta e análise de serapilheira aplicados à ciclagem de nutrientes. Floresta e Ambiente 2012; 2(2): 1-18.

Selle GL. Ciclagem de nutrientes em ecossistemas florestais. Biociência Jornal 2007; 23(4): 29-39.

Souza JA, Davide AC. Deposição de serrapilheira e nutrientes em uma mata não minerada e em plantações de bracatinga (Mimosa scabrella) e de eucalipto (Eucalyptus saligna) em áreas de mineração de bauxita. Cerne 2001; 7(1): 101-113.

Vallejo LR. A influência do Litter na distribuição das águas pluviais [dissertação]. Rio de Janeiro: Universidade Federal do Rio de Janeiro; 1982.
Vieira HT Jr, Moraes JM, Schobbenhaus C, Paula TLF. Geoparque Chapada dos Guimarães - MT: proposta. Goiânia: CPRM, 2011 [citado em 2014 mar. 01]. 60 p. Disponível em: http://www.cprm.gov.br/geoecoturismo/ geoparques/chapada/caracterizacaofisica.html

Vital ART, Guerrini IA, Franken WK, Fonseca RCB. Produção de serapilheira e ciclagem de nutrientes de uma Floresta Estacional Semidecidual em zona ripária. Revista Árvore 2004; 8: 793-800.

Wolkovich EM. Nonnative grass litter enhances grazing arthropod assemblages by increasing native shrub growth. Ecology 2010; 91(3): 756-766.

Zaia FC, Gama-Rodrigues AC. Nutrient cycling and balance in eucalypt plantation systems in north of Rio de Janeiro State, Brazil. Revista Brasileira de Ciencia do Solo 2004; 28(5): 843-852. 